Relations industrielles

Industrial Relations

\title{
The Imperfect Union, A History of Corruption in American Trade Unions, by John Hutchinson, New York, E.P. Dutton \& Co. Inc., 1972,477 p.
}

\section{Gérard Dion}

\section{Volume 27, numéro 4, 1972}

URI : https://id.erudit.org/iderudit/028346ar

DOI : https://doi.org/10.7202/028346ar

Aller au sommaire du numéro

Éditeur(s)

Département des relations industrielles de l'Université Laval

ISSN

0034-379X (imprimé)

1703-8138 (numérique)

Découvrir la revue

Citer ce compte rendu

Dion, G. (1972). Compte rendu de [The Imperfect Union, A History of Corruption in American Trade Unions, by John Hutchinson, New York, E.P. Dutton \& Co. Inc., 1972, 477 p.] Relations industrielles / Industrial Relations, 27(4), 791-792. https://doi.org/10.7202/028346ar

Tous droits réservés (C Département des relations industrielles de l'Université Laval, 1972
Ce document est protégé par la loi sur le droit d'auteur. L'utilisation des services d'Érudit (y compris la reproduction) est assujettie à sa politique d'utilisation que vous pouvez consulter en ligne.

https://apropos.erudit.org/fr/usagers/politique-dutilisation/ 
procédure de travail. C'est donc un instrument essentiellement 《didactique », façonné pour une fin d'apprentissage, et non un instrument de recherche empirique. J'aurais aimé discuter de la valeur pratique du questionnaire, mais je n'ai pas eu l'occasion de l'utiliser.

\section{Laurent BELANGER}

Economic Theory of Teams, by Jacob Marschak and Roy Radner, New Haven and London, Yale University Press, 1972, X, 345 p.

Marschak est le père de la théorie économique des «équipes 》 (teams). Avec l'aide de Radner il nous présente une espèce de somme théorique qui n'a plus l'ingéniosité succinte de ses premiers articles sur le sujet. L'intérêt se perd dans toute la partie centrale, excessivement formelle, de ce gros livre. Les premiers chapitres et les derniers stimulent un peu plus le lecteur.

La problématique des équipes est posée de façon simple dès la préface. Ce sont des organisations dont les membres partagent les mêmes intérêts et les mêmes croyances (beliefs), mais n'ont pas le même degré d'information. Le problème qui se pose à ce type d'organisation, ou à un «organisateur » d'équipe peut alors être formulé ainsi : comment répartir les tâches de recherche, de communication et de décision parmi les membres, de façon à atteindre des résultats optimaux, du point de vue de l'organisation ou de celui de l'organisateur. Comme on le voit la perspective est celle de l'économique, définie au sens large comme la science de l'utilisation efficace, ou optimale, de ressources rares.

Dans ce qu'ils croient être une démarche de bonne méthode les auteurs commencent par s'interroger sur la décision individuelle. C'est l'occasion pour eux de présenter de façon cohérente un ensemble de concepts qui seront ensuite utilisés dans l'étude des équipes. Dès ce moment la discussion se perd trop souvent dans une formalisation excessive dont on ne voit pas trop bien l'utilité pour l'étude des problèmes précis qui ont été présentés au début du livre.

La deuxième partie est consacrée à l'étude de ces problèmes qui se posent aux équipes. On passe donc de l'individu à l'organisation, et des concepts à leur application. Comme on l'a déjà signalé, ces applications demeurent excessivement formelles, et surtout elles semblent commandées davantage par les exigences mêmes de la formalisation plutôt que par le souci de répondre à des problèmes concrets. Cette théorie des équipes court ainsi le risque de répéter l'échec de la théorie mathématique des jeux : à appliquer une théorie élaborée selon les seules exigences de la formulation mathématique on n'arrive pas à constituer vraiment les phénomènes sociaux étudiés, c'est-àdire qu'on ne fait pas de science sociale.

Toutefois les auteurs ouvrent dans les chapitres 8 et 9 une voie nouvelle, où leur analyse évite un peu mieux l'écueil qu'on vient de signaler. Ils considèrent le problème d'une équipe comme un problème de réseau de communication qu'il s'agit d'optimaliser. On pourrait croire qu'ils vont rejoindre ainsi les nombreux travaux des psychologues sociaux sur les réseaux de communication. Mais il n'en est rien. Tout au plus s'inspirent-ils des ingénieurs en communication. Pourtant on n'a qu'à lire l'excellent petit livre de Flament Réseaux de communication et structures de groupe, Paris, Dunod, 1965, qui, soit dit en passant, est aussi bon mathématicien que Marschak et Radner, pour voir tout ce que les psychologues sociaux peuvent apporter à une théorie, même économique, des réseaux.

Le spécialiste des relations industrielles ne pourra donc rien tirer de ce livre, à moins qu'il le prenne comme un divertissement mathématique. La théorie économique des vraies équipes, de négociateurs, de permanents syndicaux, de direction des ministères, etc., reste encore à construire.

Vincent LEMIEUX

The Imperfect Union, A History of Corruption in American Trade Unions, by John Hutchinsen, New York, E. P. Dutton \& Co. Inc., 1972, 477 pp.

Cet ouvrage retrace l'histoire de la corruption dans le syndicalisme américain à partir de 1890 jusqu'aux années soixante. Il analyse des cas, essaie d'expliquer l'origine de ce problème, ses causes et sa persistance.

La corruption syndicale est une question complexe pour laquelle il est assez facile de se laisser aller à des jugements subjectifs. Voulant éviter ce piège, l'auteur s'est arrêté à un critère: "Trade unionism should not be employed as an 
instrument of commercial profit. I have therefore chosen to regard corruption as the use of union power for private enrichment by anyone. There are other forms of the disease, but I am concerned with them here only as they contribute to the unsanctioned pursuit of money. This is a selective standard; but it seems to be a pertinent one, quite sufficient for one volume $\gg$.

Après un bref prologue dans lequel sont rappelées la morale des affaires en vogue à la fin du siècle dernier et la philosophie de la FAT, l'auteur aborde la corruption dans l'industrie de la construction. Il étudie ensuite plusieurs cas de racketérisme dans quelques villes et quelques industries et rapporte les grandes enquêtes menées par le Congrès américain. Enfin, dans une dernière partie il expose l'attitude des centrales syndicales, la FAT, la COI et la FAT-COI vis-à-vis de la corruption ainsi que les mesures législatives qui ont été adoptées et, il consacre un chapitre à une analyse pénétrante de ce qu'il appelle l'anatomie de la corruption.

Cet ouvrage est bien documenté. L'auteur a pris la peine de sa référer uniquement à des sources fiables. Ses jugements sont pondérés. Il sait éviter les exagérations et les généralisations sans passer l'éponge sur ce mal réel dans le syndicalisme américain. Le problème de la corruption syndicale est fondamentalement un problème de la société dans lequel il existe. A chaque fois que l'on rencontre des dirigeants syndicaux corrompus il y a aussi des employeurs ou des politiciens complices et souvent des syndiqués indifférents. Si la législation peut apporter une contribution pour résoudre ce problème elle seule, elle est assez inefficace. Il est indispensable de changer le climat général et de pouvoir compter sur la collaboration de tous. Les statuts des grandes centrales en raison d'une décentralisation très poussée ne laissent qu'une minime marge d'action aux dirigeants sur les corps affiliés. Toutefois, ceux-ci ont toujours eu souci de la réputation du syndicalisme et ont pris des mesures visant à enrayer la corruption.

Dans l'abondante littérature qui existe sur ce sujet l'ouvrage de John Hutchinson est l'étude d'ensemble la plus complète et la plus positive.

Gérard DION
Arbitration and Collective Bargaining : Conflict Resolution in Labor Arbitration, by Paul Prasow and Edward Peters, New York, McGraw-Hill Book Co., 1970, 426 pp.

Cet ouvrage porte sur l'arbitrage des griefs, des mésententes qui surviennent au cours de l'application de la convention collective. D'une masse de décisions rendues par les arbitres et les cours de justice, les auteurs tentent de dégager quelques généralisations, une sorte de «droit commun $»$ qui peut servir de guide à ceux qui jouent actuellement le rôle d'arbitres. On retrouve évidemment dans cet ouvrage les mêmes thèmes traités par d'autres auteurs dans des livres et articles de revues. Cependant, ce volume se distingue par l'ordonnancernent de la matière en donnant une vue d'ensemble des principes qui président à l'arbitrage des griefs et en les illustrant par des cas-types qui ont marqué l'évoluticn du système aux Etats-Unis.

Un bref historique de la négociation collective, l'apparition du mécanisme d'arbitrage et les modifications qu'il a subies après la deuxième guerre constituent l'objet du premier chapitre. Au deuxième, les auteurs tentent de préciser les sources et la sphère d'autorité de l'arbitre. Tout en admettant que le pouvoir d'un arbitre est limité par les clauses qui décrivent la procédure de griefs dans chaque convention collective, ils font ressortir d'autres éléments du système dont l'arbitre peut tirer son autorité. La qualité de la preuve faite devant lui compte pour beaucoup. A cela s'ajoutent les coutumes et les pratiques des ateliers de travail.

Le chapitre III tente de replacer dans son véritable contexte la théorie des droits de gérance et de son corrollaire, la doctrine des obligations implicites qui découlent du contrat de travail. Les chapitres IV, V, VI et VII traitent des problèmes d'interprétation des termes des diverses clauses du contrat et du poids qu'on peut accorder aux usages (past practices) en vue d'éclairer la signification d'un terme ou encore donner un effet à une clause générale lorsque le contrat ne couvre pas de façon explicite un point particulier qui fait l'objet d'un grief. Le chapitre VIII examine plus en profondeur les problèmes de promotion, de mutation, de démotion en distinguant entre les mésententes portant sur l'essence mê- 